\title{
À LA RECHERCHE DE LA FORME ET DU SENS DES GESTES QUASI-LINGUISTIQUES ET SYLLINGUISTIQUES DANS LA COMMUNICATION FRANÇAISE, ITALIENNE ET POLONAISE
}

\begin{abstract}
A bstract. Szarota Beata, À la recherche de la forme et du sens des gestes quasi-linguistiques et syllinguistiques dans la communication française, italienne et polonaise [In the search of the form and sense of quasi-linguistic and sylinguistic gestures in the French Italian and Polish communication]. Studia Romanica Posnaniensia, Adam Mickiewicz University Press, Poznań, vol. XXXVII/2: 2010, pp. 85-93. ISBN 978-83-232-2189-0. ISSN 0137-2475. DOI 10.2478/v10123-010-0017-z.
\end{abstract}

The present paper is an attempt supplying proofs that gestural signs are almost like standard linguistic signs. It is claimed that language gestures exhibit numerous properties of classical linguistic signs conceived of as in the works of de Saussere. The dividing line between the two pertains, in essence, to the type of motivation between the signifier and the signified, found within gestural, but not linguistic signs. There is a conspicuous connection between the two aspects of the sign within various kinds of gestures, including volitional ones (most akin to speech acts or "la parole” of the Saussure) as well as within those which are not performed on purpose (called ,sylinguistic" gestures)

\section{INTRODUCTION}

Notre article a pour but de présenter un système de segmentation gestuelle à partir duquel on peut établir le rapport entre le signifiant et le signifié, rapport qui décide du caractère motivé des gestes.

\section{CLAS SIFICATION}

Parmi plusieurs classifications qui, au sens large, regroupent des gestes : en mouvement corrélé avec la parole et en mouvement centré sur le corps, nous retiendrons plus particulièrement celle de Bogdanka Pavelin (2002, p. 100).

Le point de départ pour sa classification était l'analyse de Jacques Cosnier qui répartit les gestes en communicatifs et en extra-communicatifs.

Les gestes communicatifs sont toujours référentiels et liés au déroulement verbal.

Les extra-communicatifs (gestes autocentrés, ludiques et de confort) n'entraînent pas d'une manière explicite et (semi)consciente le décodage de l'information. 
Ce type gestuel n'est pas orienté sur le destinataire ni sur l'acte de langage mais sur l'émetteur. Il n'a pas de fonction précise de six éléments « officiels » du système Shannon/Jakobson.

Pavelin a changé ces deux notions de base. Elle a regroupé toute la posturomimogestuelle en langagière et extra-langagière en justifiant que « [...] les termes communicatif et extra-communicatif semblent inadéquats ou en tout cas mal choisis, car tout comportement est communicatif une fois qu'il fait partie d'une communication en face-à-face » (2002, p. 101).

Les gestes langagiers sont répartis selon les fonctions qu'ils exercent en :

$\rightarrow$ quasi-lingustiques, qui peuvent être produits sans parole concomitante,

$\rightarrow$ syllinguistiques, qui sont obligatoirement en co-occurrence avec la production verbale,

$\rightarrow$ synchronisateurs, qui concernent la strate interactionnelle d'un événement langagier.

Il faut également prendre en considération le côté pragmatique de l'énoncé qui peut modifier la fonctionalité des gestes. Les mêmes formes gestuelles peuvent appartenir à plusieurs catégories à la fois.

Puisque nous nous concentrons sur l'aspect de la communication consciente et volontaire, nous avons restreint le champ des recherches aux gestes quasi-linguistiques et aux gestes syllinguistiques-illustratifs dont l'analyse des rapports entre la forme et le sens est peu équivoque.

\section{LES QUASI-LINGUISTIQUES}

Les gestes quasi-linguistiques sont explicites, conventionnels et socialement codifiés. Ils peuvent être produits en concomitance avec la parole ou indépendamment de l'énoncé verbal. Ils représentent une idée traduisible par un mot ou par une phrase. Au niveau sémantique, les gestes quasi-linguistiques relèvent des particularités parallèles aux signes linguistiques notamment; variabilité diachronique et conventionalité.

Par la variabilité on comprend deux spécificités à première vue contradictoires : immutabilité et mutabilité du signe.

Le signifiant par rapport à une communauté linguistique n'est pas librement choisi, il est toujours imposé. Le geste-emblème autant que le signe linguistique constitue « un héritage du siècle précédent, une convention admise par les membres d'une communauté linguistique et transmise aux membres de la génération suivante » (Saussure, 2002, p. 95). Il en résulte qu'il doit être compris, accepté et conservé par tous.

Toutefois le temps qui d'un côté assure la continuité de la langue (c'est-à-dire l'ensemble de facteurs communicatifs : verbals, vocaux et mimogestuels) a un autre effet, celui d'altérer le signe. Ces changements peuvent aboutir à un déplacement total ou partiel du rapport entre le signifiant et le signifié. C'est ce que prouvent les exemples qui suivent. 

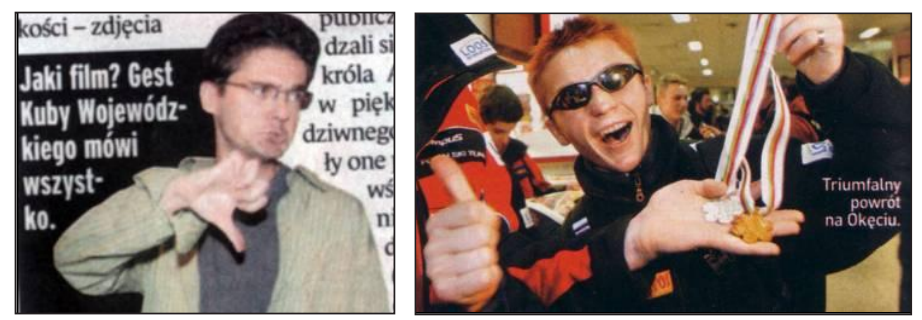

Dans l'antiquité, les Romain, en tournant leur pouce vers le bas ou vers le haut ${ }^{1}$ réclamaient ou non la mort du gladiateur. Aujourd'hui, ce geste exprime un sentiment de défaite ou de joie.

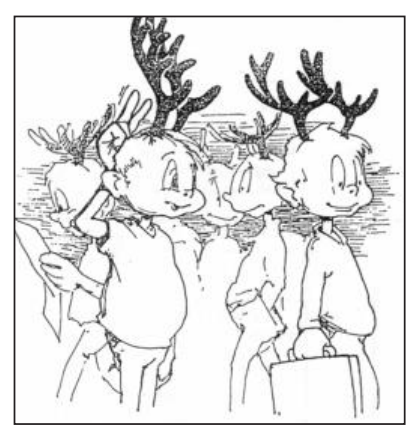

De même, les cornes symbolisaient la force et le courage. Hans Biedermann (2003, p. 308) note: « jusqu'au Moyen Âge ornaient les heaumes des guerrieurs, par exemple : des Gaulois et des Germains ». Les cornes sont donc un symbole belliqueux. Alors qu'aujourd'hui, dans les cultures française, italienne et polonaise elles ont une connotation sexuelle : l'injure exprimant l'adultère.

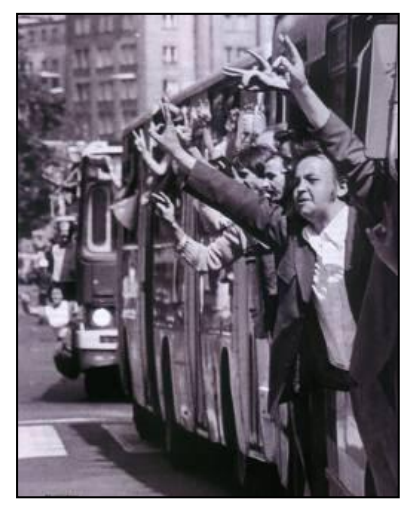

Un déplacement partiel du même genre s'observe pour le geste imitant le « V » de victoire utilisé par exemple par Winston Churchill dans les années 40.

En effet, au début des années quatre-vingts, les grévistes des chantiers navals de Gdańsk ont repris ce geste pour exprimer leur opposition au régime communiste. Puis ce geste est devenu le symbole des syndicats ouvriers « Solidarność ». C'est avant tout à cela que les Polonais associent ce geste, et pas au symbole de la victoire.

Chaque geste a également un trait propre au système non-verbal : le caractère motivé, entendu comme «l'ensemble des facteurs conscients ou semi-conscients qui conduisent un individu à avoir un comportement déterminé dans le domaine linguistique » (Dubois, 1973, p. 328). Cela constitue une analogie explicable entre l'aspect physique d'un geste et sa signification tout en différent d'une culture à l'autre.

\footnotetext{
${ }^{1}$ Munari $(2004$, p. 21$):$ « [...] le pouce tourné vers le bas : la morte»; Kopaliński (1985, p. 479) : « [...] żądanie dobicia rannego gladiatora w rzymskim cyrku wyrażano poprzez podniesienia kciuka w górę i, być może, kręcenie nim. Obrócenie kciuka ku dołowi oznaczało życzenie ułaskawienia ».
} 
Le caractère motivé peut être plus ou moins évident d'où les interprétations faites par l'interlocuteur s'expliquent soit par une assimilation à un signe déjà connu, soit par une recherche déductive de motivation.

L'iconicité des gestes quasi-linguistiques reste très variable : des gestes qui miment toute l'action jusqu'aux ceux fortement codés et symboliques. On peut examiner la relation entre la forme et le sens des gestes quasi-linguistiques sur le plan « physiologique » et « lexicologique».

Les quasi-linguistiques motivés « physiologiquement » reflètent le fonctionnement de l'organisme.

Certains gestes illustrent toute l'action :

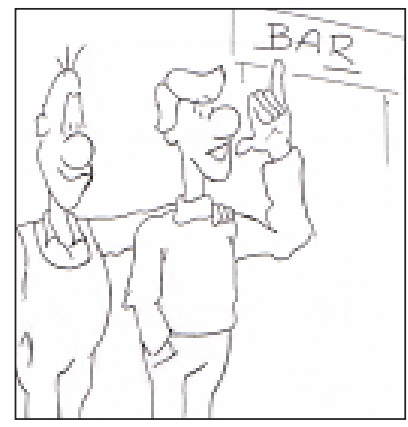

- boire : la tête en arrière, la main refermée (sur une bouteille imaginaire) ;

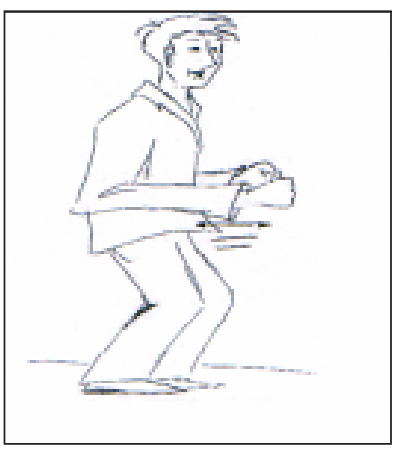

- gestes érotiques : mouvoir en avant et en arrière les mains et les hanches.

Le plus souvent, ils remplissent une fonction référentielle. Ils nous informent sur l'état ou l'action éxécutée.

Les gestes de pointage, eux, parfois accompagnés d'une mimique expressive, s'orientent sur un élément particulier, un organe. Ils peuvent avoir une fonction conative (entendre : l'index désignant l'oreille) soit référentielle (exprimer la stupidité : l'index qui vrille sur la tempe).

Le groupe suivant des gestes quasi-linguistiques constitue celui dont le rapport entre le signifié et le signifiant représente le symbolisme conventionnel. 
Le caractère motivé de ces gestes peut s'avérer plus ou moins obscur car ils ont pour but d'accompagner ou de se substituer à des expressions idiomatiques. Des personnes de langues différentes peuvent comprendre ces gestes s'ils ont des équivalents verbaux dans leur langue.
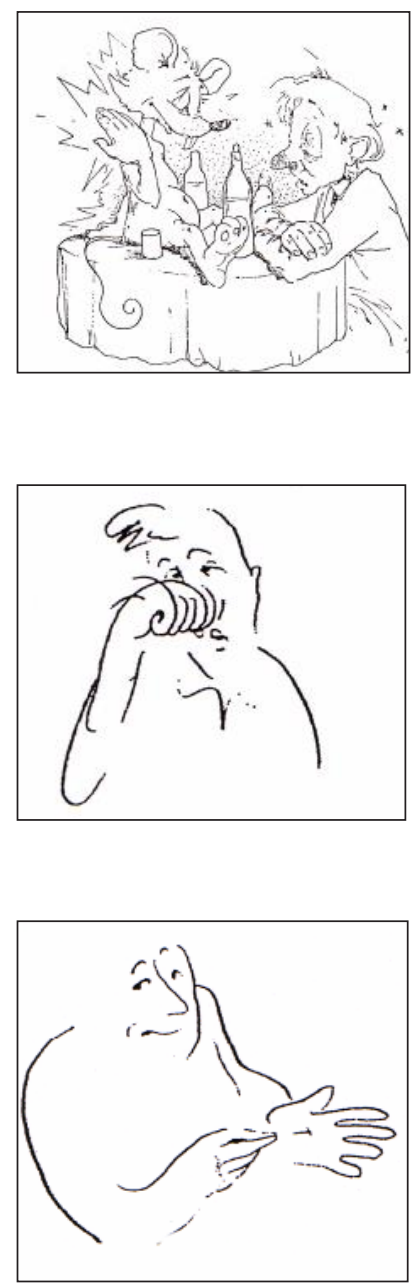

Par exemple : le geste polonais de se toucher plusieurs fois le cou avec la main tendue horizontalement, exprime l'ivresse ou l'invitation à boire. La forme gestuelle associe à l'expression idiomatique « nie wylewać za kołnierz » (« ne pas verser » de l'alcool en arrière, sous le col). Jan Bystroń note qu'au XVI-XVII ${ }^{\text {ième }}$ siècle pendant plusieurs fêtes des hobereaux, il arrivait que les convives complétement soûls ne réussisaient pas à porter le verre à leur bouche et qu'ils versaient du vin derrière eux.

Les Français expriment cette même idée par un geste de la main. La main, les doigts enroulés sur eux-mêmes autour du pouce exécute une rotation autour du nez.

L'expression équivalente à ce geste est « avoir un coup dans le nez » ou « être rond comme une queue de pelle ».

Nous recourons aussi à un geste signifiant: « avoir un poil dans la main » pour indiquer une personne paresseuse.

Le pouce et l'index droits, réunis en pointe, s'éloignent lentement du milieu de la paume gauche, en tirant et en mesurant ainsi un poil imaginaire qui aurait pu pousser sur la main d'une personne qui ne travaille pas.

\section{LES SYLLINGUISTIQUES}

Les syllinguistiques constituent un groupe des gestes dépendant de l'énoncé verbal. On en distingue trois sous-types: phonogènes, coverbaux et synchronisateurs. Ce qui nous intéresse le plus ce sont des coverbaux-illustratifs. Ils réfèrent au contenu 
verbal soit par une corrélation entre l'acte du langage et les composantes situationnelles (les gestes déictiques) soit par le contenu de l'énoncé exprimé sous forme de représentations figuratives dans l'espace gesticulatoire.

\section{a. Les déictiques}

Les déixis expriment les objets matériels ou abstraits. Elles remplissent une fonction désignative, impérative et descriptive. Le rapport entre le signifiant et le signifié est un rapport de contiguité qui nous renvoie directement au référent. Ces gestes comme tout signe-indice représentent l'idée de perception égocentrique du monde. En effet, le locuteur situe gestuellement des notions à certains endroits de l'espace en prenant son corps comme point de référence.

L'homme occidental se situe dans le temps en fonction de son déplacement de l'espace. Le temps se relève comme un axe symétrique, comme un trajet à faire.

Geneviève Calbris et Jacques Montredon (1986, p. 139) notent que le locuteur localise le présent en bas, à ses pieds, le futur est devant lui et le passé est derrière.

Ici, le système gestuel est parallèle au verbal car la conception métaphorique de l'axe temporel est repérable au niveau de la phraséologie. Les expressions françaises : « avoir toute la vie devant soi », « envisager son avénir » et « regarder en arrière » trouvent facilement les équivalents formels et sémantiques de même en polonais : «mieć życie przed sobą ", " patrzeć w przyszłość », «(nie) oglądać się za siebie » et en italien : « avere tutta la vita davanti a sé », « guardare al futuro », « gettarsi dietro le spalle le preoccupazioni ».

On peut constater un rapprochement analogique et proportionnel entre l'ampleur de l'espace occupé gestuellement, entre la rapidité des mouvements et entre la distance et la quantité du temps mesuré. Plus la distance est grande, plus l'index ou toute la main se redresse et/ou fait un geste plus lent et ample.

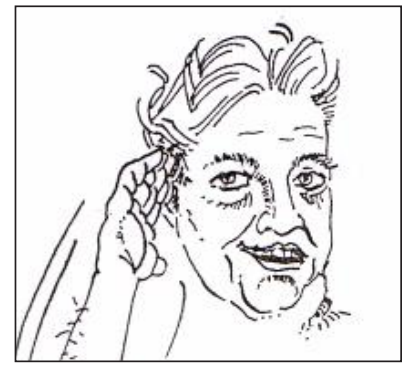

Les Napolitains expriment l'idée du jour précédent avec la main levée près de la tête et remuée vers l'épaule. 

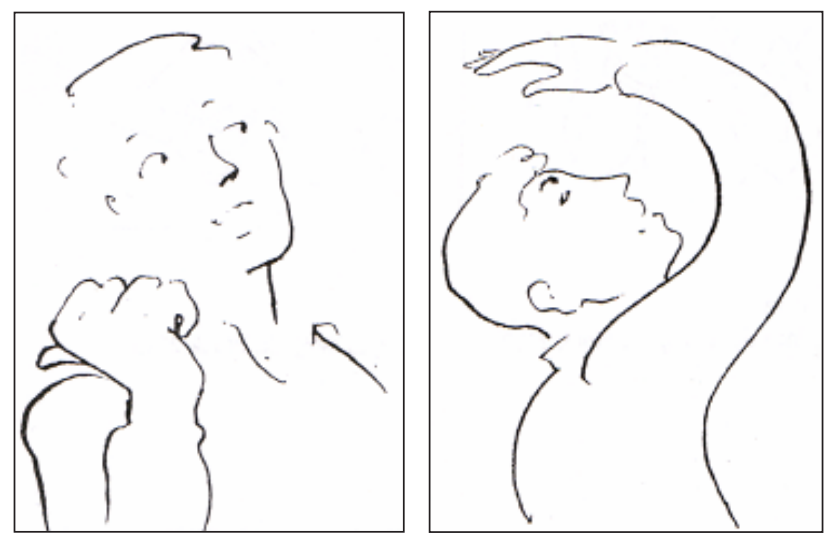

Les Français situent le passé proche juste derrière eux avec le pouce et/ou la tête tourné(s) par dessus l'épaule tandis que le mouvement plus large en arrière avec tout l'avant-bras est entendu comme passé lointain et imprécis.

L'expression polonaise « oooo, dużo czasu upłynęło » (« ooo, ça fait beacoup de temps ») est souvent accompagnée avec un large mouvement de la main dirigée en avant. Le cours du temps est représenté par un mouvement circulaire de la main droite placée à gauche pour être déplacée à droite. A titre d'exemple : le geste italien signifiant « demain, après », est exécuté avec l'indice formant des petits arcs en avant. On suppose que ce geste réfère au lever et coucher du soleil.

Le mode de localisation temporelle est partiellement arbitraire et culturel puisque c'est une conception linguistique qui détermine la manière de présenter gestuellement le sens de l'énoncé verbal. Jacques Montredon (1998, p. 149) décrit des cultures où on observe une logique opposée par rapport à la représentation du futur projeté dans le sens de la marche. « C'est ainsi qu'en toba, une langue amérindienne parlée au Chaco, en Amérique Du Sud, [...] le locuteur pour indiquer le passé, le désigne par un mouvement en avant tandis qu'il doit tourner la tête et jeter un coup d'œil en arrière pour se référer au futur, qu'il peut voir ainsi s'approcher. [...] La logique de ces positionnements est irréfutable : le passé proche est devant puisqu'on le connait, qu'on vient de le voir, tandis que le futur proche est derrière nous, encore invisible ».

On pourrait donc en conclure que notre organisation gestuelle coexiste avec l'énoncé verbal et dépend de la conception métaphorique conçue dans l'esprit.

Dans la communication courante on emploie aussi des déictiques concrets, liés aux objets et au contexte spacio-situationnel. Ils évoquent immédiatemnet un référent pour le faire voir à l'interlocuteur avec toute la main, l'index et/ou avec la tête et les yeux. L'énoncé est souvent accompagné et renforcé par des adverbes de lieu, pronoms démonstratifs, adverbes de temps. 


\section{b. Les illustratifs}

Les trois types des gestes iconiques constituent un autre sous-groupe des illustratifs. Ils représentent la relation de ressemblence entre le sens et la forme. Ils ont la même fonction : se rapporter à l'image d'un référent. Ils servent à rendre concret et visible un élément du contenu de l'énoncé verbal, de le " faire apparaître » ou bien de le «mettre en présence» dans l'espace gesticulatoire (Pavelin 2002, p. 108). La manière d'exprimer le sens est pourtant différente.

Les pictomimiques reproduisent les contours ou les qualités d'un référent. Ils sont schématisés : ils ne distinguent que les traits les plus particuliers du référent et nécessaires pour le reconnaître, par exemple :

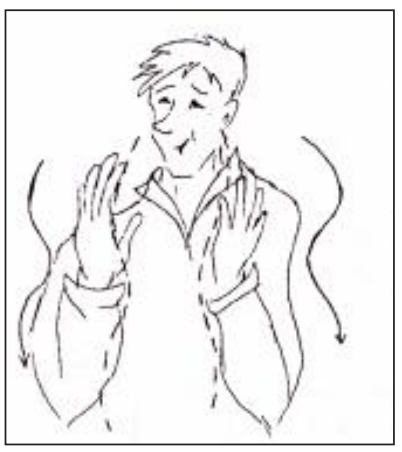

Les hommes parlant d'une femme font deux demi-cercles avec les mains. La dimension illocutoire est claire : l'enthousiasme pour les attributs féminins physiques.

L'élément gestuel de cet énoncé remplit plusieurs fonctions pragmatiques : il est redondant par rapport au verbal, il peut le préciser, l'économiser, soit au cas de divergence entre l'énoncé verbal et non-verbal connoter d'une manière juste.

Les spatiomimiques reproduisent une structure spatiale. L'énoncé : «Ils ont grimpé à l'arbre » en mimant la corde imaginaire avec les mains, est dépourvu de l'information sur la façon de réaliser l'action. La gestualité a ici une valeur complémentive de l'acte de langage.

Les kinémimiques évoquent l'action ou l'état dont on parle dans le discours verbal. Un jeune garçon raconte la dernière fête : - « Je me rappelle rien, je sais pas comment je suis descendu du taxi », et il se balance avec les mains levées devant lui. La gestualité qui accompagne cet énoncé a un rôle primordial car elle nous fait découvrir une vraie raison de l'indisposition du locuteur.

\section{CONCLUSION}

Nous avons cherché à fournir un échantillon d'exemples des comportements quasi-linguistiques et syllinguistiques dans la communication française, italienne et polonaise qui illustraient le caractère non-arbitraire et convergent entre le sens de l'énoncé 
et la forme extérieure. La motivation gestuelle peut être plus ou moins évidente et déchiffrable et elle résulte de la situation contextuelle, langagière et sociale de l'acte de communication.

\section{BIBLIOGRAPHIE}

Biedermann, H. (2003). Leksykon symboli. Warszawa: Muza SA.

Bystroń, J. (1932). Dzieje obyczajów w dawnej Polsce wiek XVI-XVIII. Warszawa: PIW.

Calbris, G., \& Montredon, J. (1986). Des gestes et des mots pour le dire. Paris : CLÉ International.

Cosnier, J. (1982). Communications et langages gestuels. In Les voies du langage: communication verbales, gestuelles et animales (pp. 255-303). Paris : Bordas.

Dubois, J. (1973). Dictionnaire de linguistique. Paris : Larousse.

Kopaliński, W. (1985). Stownik mitów i tradycji kultury. Warszawa: PIW.

Montredon, J. (1998). Comment la gestuelle a joué parfois identiquement parfois différemment pour figurer à partir de positions et d'oppositions spatiales universelles, des axes ou des sites temporels. In Oralité et gestualité. Paris : L'Harmattan.

Munari, B. (1963). Supplemento al dizionario italiano. Mantova: Corraini Editore.

Pavelin, B. (2002). Le geste à la parole. Toulouse : Presses Universitaires du Mirail.

Saussure de, F. (2002). Kurs językoznawstwa ogólnego. Warszawa: PWN.

Szarota, B. (2005). Charakterystyka i różnice interpretacyjne gestów na przykładzie komunikacji włoskiej, francuskiej i polskiej. Neofilologia, VII,175-188.

\section{RÉFÉRENCES PHOTOGRAPHIQUES}

1. Super Ekspress, 23-VII-2004, p. 21.

2. Magazyn Sportowy, 31-XII-2004, p. 10.

3. Jarząbek K. (1994). Gestykulacja i mimika. Katowice, p. 66.

4. Polityka-Rewolucja Solidarności, 8-VIII-2005, p. 46.

5. Gelabert M.J./Gifre E.M. (1990). Diccionario de gestos, Edelsa, Madrid, p. 44.

6. Wójcik E. (2002), Płock.

7. Jarząbek K. (1994). Gestykulacja i mimika. Katowice, p. 27.

8. Calbris G./Montredon J. (1986). Des gestes et des mots pour le dire. Paris, Clé International, p. 41 .

9. id. op. cit., p. 56.

10. Paura B./Sorge M. (1999). Comme te l'aggia dicere?, Moenia: Edizioni Intra, p. 62.

11. Calbris G./Montredon J. (1986). Des gestes et des mots pour le dire. Paris, Clé International, p. 140.

12. il. loc.

13. Wójcik E. (2002), Płock. 